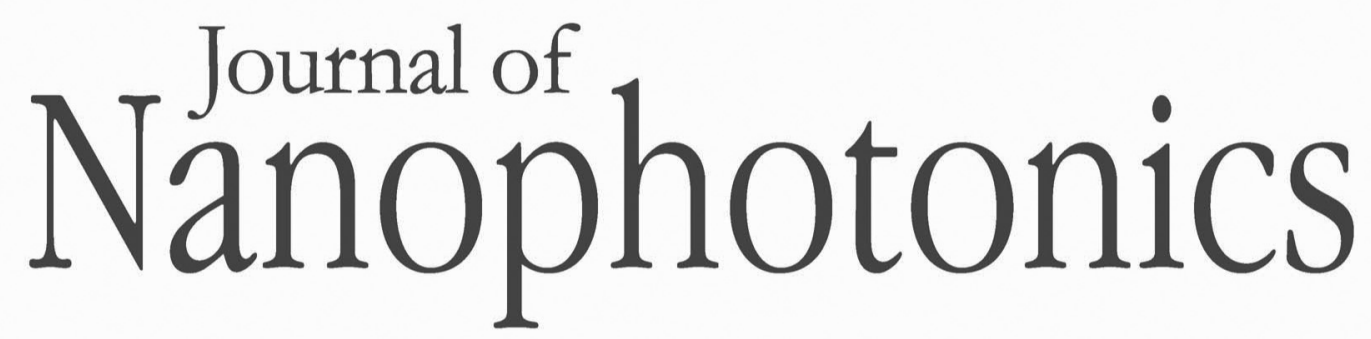

SPIEDigitalLibrary.org/jnp

\title{
Levy distribution of time delay in emission of resonantly trapped light in ferrodispersions
}

Rajesh Patel

Rasbindu Mehta 


\title{
Levy distribution of time delay in emission of resonantly trapped light in ferrodispersions
}

\author{
Rajesh Patel and Rasbindu Mehta \\ Bhavnagar University, Department of Physics, Bhavnagar 364022, India \\ rvm@bhavuni.edu
}

\begin{abstract}
Time delays in emission of resonantly trapped light in magnetite microspheres dispersed in a ferrofluid were measured for three different sphere sizes. The time delay was found to vary randomly. The effect of the size of microspheres on the probability distribution was determined. Analysis of the data showed that the statistics of the time delay is best described by a Levy distribution. () 2012 Society of Photo-Optical Instrumentation Engineers (SPIE). [DOI: 10 .1117/1.JNP.6.069503]
\end{abstract}

Keywords: Mie resonance; magnetic colloids; light trapping; magnetic tuning; Levy statistics.

Paper 12073L received Jun. 23, 2012; revised manuscript received Jul. 27, 2012; accepted for publication Jul. 27, 2012; published online Sep. 12, 2012.

\section{Introduction}

Scientific phenomena that follow Levy statistics are ubiquitous in nature. Spherical micelles of cetyltrimethyl ammonium bromide dissolved in salt water, animal foraging patterns, heart beat histograms of healthy individuals, and certain fluorescence emissions are but a few examples where Levy statistics are successfully employed. ${ }^{1-4}$ Though many papers on the numerical study of transport based on Levy flights are available, experimental work on the transport is limited. Recently it has been observed that certain optical scattering phenomena also obey Levy statistics. ${ }^{5,6}$ It has been shown that, in a random amplifying medium using a dye-scatterer system, the intensity fluctuations of emitted waves follow Levy statistics. ${ }^{5}$ Another typical example is photonic glass, ${ }^{6}$ which consists of a random arrangement of glass microspheres. Another ingredient are titanium dioxide $\left(\mathrm{TiO}_{2}\right)$ nanoparticles suspended in sodium silicate. $\mathrm{TiO}_{2}$ has a high refractive index and its number distribution was adjusted so that a single scattering event takes place between two successive glass spheres. Consequently the step length between successive scattering events will be controlled by the variation of the local density of the glass microspheres whose size distribution is selected to follow a power law distribution. Since there is no refractive index contrast between the microspheres and the host glass, they do not play a role in scattering events; their part is to modify the density of scattering elements. Selecting a precise size distribution is critical in the preparation of photonic glass. For such a system it is shown that the total transmission follows Levy statistics. ${ }^{6}$ We describe another medium where externally applied static magnetic field controls scattering events and refractive index mismatch between the scatterers and the medium play a pivotal role in the Levy transport.

Recently we synthesized a novel ferrimagnetic magnetic dispersion in which micron-sized magnetite spheres are stably dispersed in a ferrofluid. ${ }^{7}$ Composed of a large number of nanomagnetic particles of magnetite, the ferrofluid can be considered as a quasi-continuous medium ${ }^{8}$ and its refractive index is known to be controlled by externally applied magnetic field. ${ }^{9,10}$ The number density of micron-sized magnetite spheres is selected such that a Mie scattering masks the weak Rayleigh scattering due to nanomagnetic particles of ferrofluid. Under the influence of the external field, the refractive index contrast between the Mie spheres and the host ferrofluid medium can be varied by varying the field. At a critical field refractive index contrast, we assume the value that leads to Mie resonance in the system. ${ }^{11}$ This induced resonance has resulted in several intriguing, as well as useful, phenomena1. ${ }^{12-14}$ Most intriguing is the experimental evidence of the trapping and emission of light when a linearly polarized monochromatic light, with

0091-3286/2012/\$25.00 (C) 2012 SPIE 
its E-vector (direction of vibrations of electric field of the incident light) perpendicular to the applied transverse static magnetic field, is incident on the dispersion. ${ }^{12}$ Upon increasing the field, it was observed that at a critical field $\left(\mathrm{H}_{c 1}\right)$ scattered as well as transmitted light from the sample disappears and reappears when the field reaches another critical value $\left(\mathrm{H}_{c 2}\right) \cdot{ }^{12}$ At a field between $\mathrm{H}_{c 1}$ and $\mathrm{H}_{c 2}$, the sample was exposed to the incident light for some time $(t)$ and then the light shutter was first closed and subsequently the field was switched off. Within a very short interval of time $\left(\tau_{d}\right)$ a flash of light having the same characteristics (frequency, bandwidth and polarization) as that of the incident beam was observed. This suggests that light is trapped and stored at the critical field in the medium and is released after the removal of the field. Spectral characteristics of the phenomenon were also studied and reported in an earlier paper. ${ }^{13}$ Time delay $\left(\tau_{d}\right)$ was found to vary randomly in successive measurements. ${ }^{14}$ We show that this random behavior of $\tau_{d}$ is best described by Levy distribution.

\section{Experimental}

Microspheres of magnetite $\left(\mathrm{Fe}_{3} \mathrm{O}_{4}\right)$ are coated with oleic acid and suspended in the ferrofluid, which was a colloidal dispersion of nonmagnetic particles of magnetite in kerosene. The microspheres, being multidomain, have zero magnetization at zero fields. On the other hand, the nanosized particles are single-domain and hence their domain magnetization at zero fields is saturated. Consequently, they behave as tiny magnets stably dispersed in a liquid. In absence of a field, magnetic moments of these particles are randomly oriented and the system resembles a paramagnetic gas. Since the magnetic moment of particles is much larger than that of molecules of a paramagnetic gas, a ferrofluid is also referred as a superparamagnetic fluid. However, this superparamagnetic nature is different from that of Neel's superparamagnetism. ${ }^{8}$ Under stationary conditions a ferrofluid performs as a quasi-continuous medium and its properties, such as density, viscosity, and refractive index depend upon an applied static magnetic field. For a dilute ferrofluid, the field dependence of these physical properties is derived using Langevin theory of noninteracting particles. ${ }^{8}$ Since the size of microspheres are very large compared to the size of nanomagnets, light scattering will be dominated by these larger-sized particles and the scattered radiation will be confined in the forward, as well as the backward, directions. ${ }^{15}$ The field-induced variation of refractive index of a ferrofluid $\left(m_{f}\right)$ is given by

$$
m_{f}=m_{\infty} L(\xi)+m_{0} .
$$

Here, $L(\xi)$ is Langevin function given by $L(\xi)=\operatorname{coth}(\xi)-1 / \xi$, and $\xi(\mu H / K T)$ is the dimensionless field parameter; $\mu, H, K$ and $T$ are respectively dipole moment of the particles, applied magnetic field, Boltzmann constant and absolute temperature.

Hence, relative refractive index $m_{s}$ of microspheres with respect to ferrofluid $\left(m_{s} / m_{f}\right)$ will also be a function of an applied field. In other words, the field provides tunable mismatch and at a critical field resonance will be induced.

A detailed procedure for preparation of samples of ferrodispersion, as well as the experimental set-up, is described in earlier papers. ${ }^{12-14}$ Three samples comprising of $0.5,1$, and $3 \mu \mathrm{m}$-sized magnetite spheres were selected for the study. An electromechanical shutter controlled the exposure time of a linearly polarized light beam from a continuous wave helium neon ( $\mathrm{CW} \mathrm{He}-\mathrm{Ne})$ laser. The light from the shutter was incident on a beamsplitter to form two identical beams, with one beam used as a reference beam falling on a reflector while the reflected beam passed through a polaroid to minimize the intensity. Another beam was incident on a rectangular glass cell, which was filled with the sample and kept between the poles of a small electromagnet. A constant current source energized the magnet and a Hall probe ascertained uniformity of the field between the pole gaps. Two matched photodiodes detected intensities of two beams and a storage oscilloscope recorded them. A PC monitored the switching actions of the shutter and the electromagnet. The electric-field vector of the incident light, the propagation vector, and the magnetic field direction were oriented perpendicular to each other. ${ }^{16}$ The field was increased gradually until the intensity of the light beam emerged from the sample cell reduced to zero. At this field the sample was exposed to the light beam for a fixed time (six seconds). Then the light shutter was closed and the field was switched off immediately. The flash emitted from 
the sample was recorded on a storage oscilloscope. There was a finite time delay between switching off the field and the emission of the flash. This experiment was repeated 100 times for each of the three samples with the analyzed results presented in the following section.

\section{Result and Discussion}

The $\tau_{d}$ for 100 consecutive observations for a $3 \mu \mathrm{m}$ particle is shown in Fig. 1. It varies randomly from 30 to 230 milliseconds. Similar data were recorded for the other two particle sizes. Probability density distribution curves of $\tau_{d}$ for the three samples are plotted in Fig. 2, which shows that these curves have long tails. The tail length decreases as the size of the particles increase. It is known that a long tail is characteristic of Levy distribution.

Levy statistics are characterized by Levy flights and/or Levy walks. Both have a broad distribution for which a second moment does not exist. In case of a Levy flight, one may assume that the velocity will be proportional to the step length ${ }^{17,18}$ hence the time of travel is proportional to the number of steps. Consequently, for Levy flights the mean square displacement does not exist as a function of time. On the other hand, Levy walks are characterized by a constant velocity and an independent step length. Hence the time of travel will be proportional to the total length. Consequently, for Levy walks the mean square displacement exists as a nonlinear function of time. This property makes Levy walks a convenient parameter for analysis. When experiments are performed under static conditions, there will be no difference between Levy flights or Levy walks. Since time evolution of Levy flights is simple as compared to Levy walks, the former is usually used to analyze statistical data. ${ }^{1-6}$ As stated above, photonic glass is an ideal experimental material to tune Levy flights in a controlled way. This is made possible

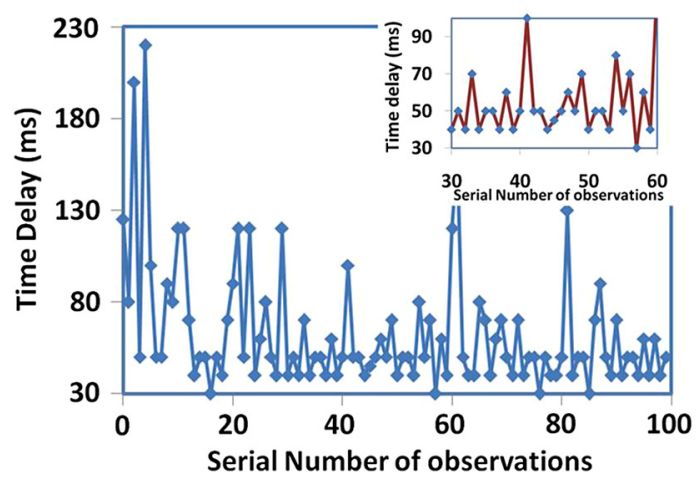

Fig. 1 Variation of time delay for 100 independent observations for three micron magnetic sphere dispersed in ferrofluid. The inset shows an enlarged view of the short and long random pulses of delay time as a function of number of observations.

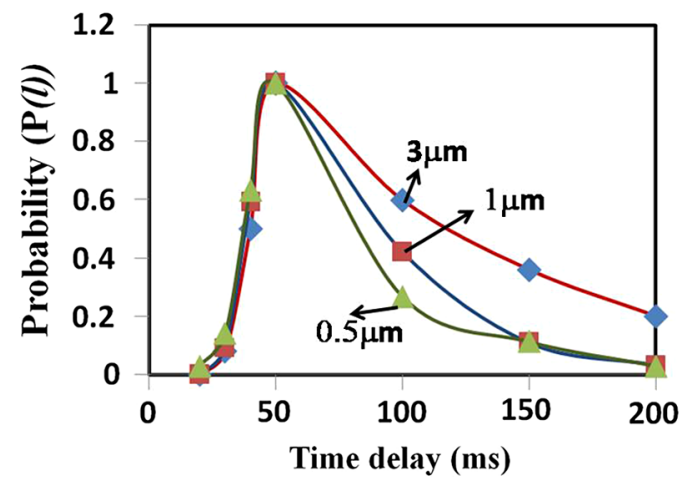

Fig. 2 Probability density distribution curves of the time delay for the three different sizes of microspheres. 
by precisely selecting size distribution of the microsphere. ${ }^{6}$ Ferrodispersion is also composed of microspheres, but here Mie scattering is tuned by a magnetically controlled mismatch of a refractive index between magnetizable microspheres and surrounding ferrofluid medium. In photonic glass there was no mismatch of refractive index between glass microspheres and the surrounding glassy medium.

Mie resonances in scattering systems can arise due to refractive index mismatch, shape or size, and are called the morphology-dependent resonance (MDR). ${ }^{19}$ Light trapping in such systems is also observed ${ }^{20,21}$ and the $Q$ factor here is found to be very large $\left(>10^{8}\right)$. Considering all the above aspects we infer that the field induced disappearance of light is due to the trapping of light while switching off the field results in a release of the trapped light. Magnetizing the Mie spheres and having the same size aligned in the same direction upon applying the field also helps. Consequently, the emission of light by all the Mie spheres may occur in the same direction. Note that light remains resonantly trapped within the dispersion as long as a magnetic field is on and emission takes place only after the field is switched off. In other words, the applied magnetic field controls the storage as well as the release of light.

The large delay time observed in the present case may be due to the storage of very large internal energy at resonance. ${ }^{4,22}$ The time delay in the case of resonant Mie scattering is given by

$$
\tau_{d}(\lambda)=[W(\lambda, S)] \times\left(\sigma_{\text {scat }} c_{0}\right)^{-1} .
$$

Here, $W(\lambda, S)$ is the energy within the scatterer $S$ at wavelength $\lambda ; \sigma_{\text {scat }}$ and $c_{0}$ are the scattering cross section and the free space velocity of light. It has been shown that at resonance this internal energy will be very large, resulting into very large $\tau_{d}{ }^{22}$ When the applied magnetic field is switched off, the system returns to its original disordered state. Usually Brownian rotation of particles restores the thermal equilibrium. In the case of a system, having "memory" effects and/or long-range correlations, such as nonthermal noise, may also occur. ${ }^{23}$ Tails of distribution curves of delay time follow power law $^{24}$

$$
P(l) \approx l^{-\mu},
$$

where $P(l)$ is the probability distribution of step or flight length and exponent $\mu$ lies between 1 and 3. If $\mu>3$ then the distribution will have Gaussian behavior, while values of $\mu \leq 1$ do not correspond to normal probability distributions. If one assumes that a jump of length $l_{j}$ takes time ' $t$ ' then the mean square displacement of Levy walks for $2<\mu<3$ will be given by $t^{4-\mu}$ and as $t^{2}$ for $\mu<2$. Strictly speaking, in Levy flights each jump regardless of its size takes one unit of time. Such behavior corresponds to anomalous super-diffusive motion. ${ }^{22,24-27}$ In the present study, the coefficient $\mu$ for 3, 1 and $0.5 \mu \mathrm{m}$-sized microspheres determined from plots of log $P_{N}(1)$ versus time delay are found to be $1.89,2$, and 2.6 , respectively, i.e., lies between 1 and 3 (Fig. 3). Hence, emission times of resonantly trapped light in each case may be described by Levy distribution.

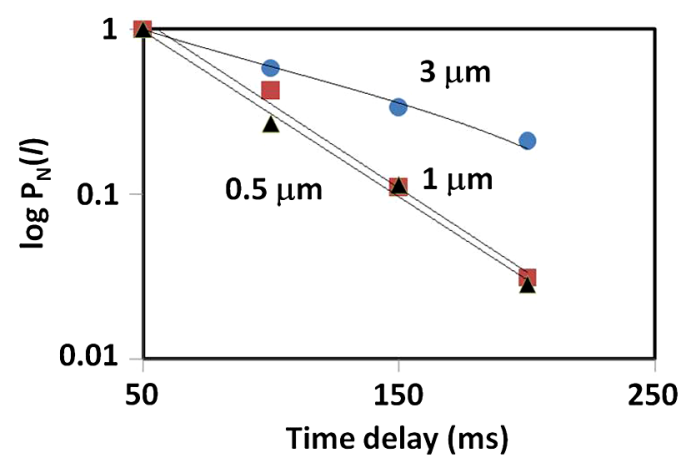

Fig. 3 Plots of $\log P_{N}(I)$ against time delay. Exponents for 3, 1, and $0.5 \mu \mathrm{m}$ are respectively 1.89, 2 , and 2.6 . 


\section{Conclusion}

In summary, we have shown that the ferrodispersion dispersion serves as a novel optical material to study Levy flight. Fluctuations in time delay in emission of resonantly trapped light in microspheres of magnetite obey Levy distribution. The probability distribution depends on the microspheres' size. Since synthesis of the ferrodispersion is easy and the technique to tune Mie resonance is simple this present work demonstrates an alternative technique to study Levy flights.

\section{Acknowledgments}

The authors wish to thank Dr. B. Chudasama and Mr. H. Desai for their help in the experimental work. They are also thankful to professors Hema Ramchandran and N. Kumar for their helpful suggestions. Rasbindu Mehta thanks the Department of Science and Technology for the Ramanna Fellowship and Rajesh Patel is grateful to the Gujarat Council on Science and Technology (GUJCOST) for the financial support of MRP-201346.

\section{References}

1. A. Ott et al., "Anomalous diffusion in living polymers: a genuine Levy flight," Phys. Rev. Lett. 65(17), 2201-2204 (1990), http://dx.doi.org/10.1103/PhysRevLett.65.2201.

2. K. Peng et al., "Long-range anticorrelations and non-Gaussian behavior of the heartbeat," Phys. Rev. Lett. 70(9), 1343-1346 (1993), http://dx.doi.org/10.1103/PhysRevLett.70.1343.

3. A. R. Bizzarri and S. Cannistraro, "Statistical analysis of intensity fluctuations in single molecule SERS spectra," Phys. Chem. Chem. Phys. 9(39), 5315-5319 (2007), http://dx .doi.org/10.1039/b706008d.

4. M. F.Shlesinger, G. Zaslavsky, and U. Frisch, Eds., Levy Flights and Related Topics in Physics, Springer, Berlin, Germany (1995).

5. D. Sharma, H. Ramchandran, and N. Kumar, "Levy statistics of emission from a novel random amplifying medium: an optical realization of the Arrhenius cascade," Opt. Lett. 31(12), 1806-1808 (2006), http://dx.doi.org/10.1364/OL.31.001806.

6. P. Barthelemy, J. Bertolotti, and D. S. Wiersma, "A Levy flight for light," Nature 453, 495-498 (2008), http://dx.doi.org/10.1038/nature06948.

7. R. V. Mehta et al., "Experimental evidence of zero forward scattering by magnetic spheres," Phys. Rev. Lett. 96(12), 127402 (2006), http://dx.doi.org/10.1103/PhysRevLett.96.127402.

8. R. E. Rosensweig, Ferrohydrodynamics, Dover, New York (1997).

9. Y. F. Chen et al., "Thermal effect on the field-dependent refractive index of the magnetic fluid film," Appl. Phys. Lett. 82(20), 3481-3484 (2003), http://dx.doi.org/10.1063/1 .1576292 .

10. T. Liu et al., "Measurement of the magnetic field dependent refractive index of magnetic fluid in bulk," Chinese Opt. Lett. 6(3), 195-197 (2008), http://dx.doi.org/10.3788/COL.

11. H. Bhatt, R. Patel, and R. V. Mehta, "Magnetically induced Mie resonance in a magnetic sphere suspended in a ferrofluid," J. Opt. Soc. Am. A 27(4), 873-877 (2010), http://dx.doi .org/10.1364/JOSAA.27.000873.

12. R. V. Mehta et al., "Experimental investigation of magnetically induced unusual emission of light from a ferrodispersion," Opt. Lett. 33(17), 1987-1989 (2008), http://dx.doi.org/10 .1364/OL.33.001987.

13. R. Patel and R. V. Mehta, "Experimental investigations on magnetically induced photonic band gap in ferrodispersions," Eur. Phys. J. Appl. Phys. 52(3), 30702-30709 (2010), http:// dx.doi.org/10.1051/epjap/2010152.

14. R. Patel and R. V. Mehta, "Ferrodispersion: a promising candidate for an optical capacitor," Appl. Opt. 50(31), G17-G22 (2011), http://dx.doi.org/10.1364/AO.50.000G17.

15. H. C. Van de Hulst, Light Scattering by Small Particles, Dover, New York (1980).

16. R. V. Mehta, M. J. Dave, and J. N. Desai, "Application of bimagnetic rotation," Appl. Opt. 10(12), 2786 (1971), http://dx.doi.org/10.1364/AO.10.002786. 
17. S. Lepri et al., "Statistical regimes of random laser fluctuations," Phys. Rev. A 75(6), 063820 (2007), http://dx.doi.org/10.1103/PhysRevA.75.063820.

18. S. V. Buldyrev et al., "Average time spent by Levy flights and walks on an interval with absorbing boundaries," Phys. Rev. E: Stat., non linear, Soft Matter Phys. 64(4), 041108 (2001), http://dx.doi.org/10.1103/PhysRevE.64.041108.

19. H. M. Tzeng et al., "Laser emission from individual droplets at wavelengths corresponding to morphology-dependent resonances," Opt. Lett. 9(7), 499-501 (1984), http://dx.doi.org/ 10.1364/OL.9.000499.

20. H. Ikari et al., "Fluorescence MDR features of Eu3 + doped sol-gel $\mathrm{TiO}_{2}$ hydrate microspheres," Opt. Mater. 30(8), 1323-1326 (2008), http://dx.doi.org/10.1016/j.optmat.2007.06 .018 .

21. T. Takahashi et al., " Morphology dependent resonant lasing of a dye-doped microsphere prepared by non-linear optical material," Thin Solid Films 331(1-2), 298-302 (1998), http:// dx.doi.org/10.1016/S0040-6090(98)00934-1.

22. A. Lagendijk and B. A. Van Tiggelen, "Resonant multiple scattering of light," Phys. Rep. 270(3), 143-215 (1996), http://dx.doi.org/10.1016/0370-1573(95)00065-8.

23. M. Osaci, C. Abrudean, and A. Berdie, "Relaxation times in magnetic nanoparticles system and memory effect and memory effects," Acta Phys. Pol. A 112(6), 1203-1212 (2007).

24. G. M. Viswanathan et al., "Levy fights in random searches," Physica A 282(1-2), 1-12 (2000), http://dx.doi.org/10.1016/S0378-4371(00)00071-6.

25. E. Barkai and J. Klafter, "Anomalous diffusion in the strong scattering limit: a Levy walk approach," Lect. Notes Phys. 511, 373-393 (1998), http://dx.doi.org/10.1007/BFb0106949.

26. J. W. Hauss and K. W. Kehr, "Diffusion in regular and disordered lattices," Phys. Rep. 150(5-6), 263-406 (1987), http://dx.doi.org/10.1016/0370-1573(87)90005-6.

27. R. Metzler and J. Klafter, "The random walks: guide to anomalous diffusion: a fractional dynamics approach,” Phys. Rep. 339(1), 1-77 (2000), http://dx.doi.org/10.1016/S0370 -1573(00)00070-3.

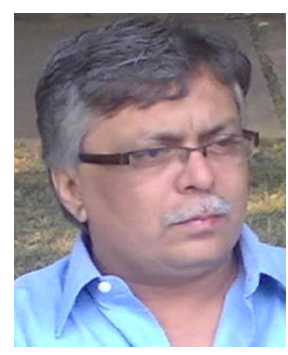

Rajesh Patel obtained his bachelor, master, and doctorate degrees from Department of Physics, Bhavnagar University, Bhavnagar, India. He is working as assistant professor at Department of Physics, Bhavnagar. His work interests are in soft matter physics, magnetic nanofluids, magnetic nano particles, rheology, and magneto-optics of magnetic fluids. He has published more than 40 papers in peer reviewed journals, and recently he has been awarded Fulbright-Nehru Senior Research Fellowship for the year 2011 to 2012 by United States India Education Foundation.

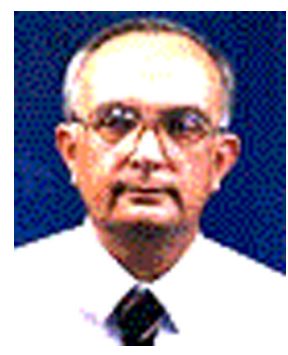

Rasbindu Mehta obtained his bachelor, master, and doctorate degrees from Gujarat University, Ahemdabad, India. From 1963 to 2002 he has served in various academic institutes and was superannuated as professor of physics at Bhavnagar University. Soon after superannuation, he was selected as CSIR Emeritus Scientist. During 2006 to 2009 he was awarded Ramanna Fellowship. He is pioneer in introducing science and technology of ferrofluids in India. He has published 125 papers and three patents. He is founder and president of Indian Society of Magnetic Fluid Research, and is a member of International Steering Committee of International Conferences on Magnetic Fluids. 